intake and HGS after intervention, readmissions and deaths were assessed at 4 months.

Results 47 and 31 patients were identified in cycle 1 and 2 respectively. A Malnutrition Universal Screening Tool (MUST) was completed in $81 \%$ of cycle 1 patients. $47 \%$ did not trigger a dietetic referral on MUST (44\% medium risk and 33\% high risk for malnutrition). All cycle 2 patients had a dietetic referral via the INP (26\% medium risk and 71\% high risk for malnutrition) and received dietary education with $77 \%$ requiring oral supplements and 10\% nasogastric feeding. At followup cycle 2 patients met higher caloric and protein requirements (average increase by $46 \%$ and $57 \%$ respectively vs $26 \%$ and $31 \%$ in cycle 1). HGS was measured in $74 \%$ in cycle 2 and $2 \%$ in cycle 1 . Average HGS was $15.9 \mathrm{~kg}$ (cycle 2) and improved by $9 \%$ on reassessment. There was a $12 \%$ reduction in hospital readmissions in cycle 2 compared to $7 \%$ increase in cycle 1 with similar mortality at 4 months.

Conclusion MUST inadequately identifies cirrhotic patients at risk of malnutrition. $\mathrm{CP}$ and BMI appear more accurate. A dedicated dietetic team and the INP enable early patient identification, thorough nutritional assessment and intervention, improving patient compliance and sarcopenia. Hospital readmission rates reduced over 4 months despite a higher proportion of high risk patients in cycle 2 vs cycle 1.12 month follow-up data will assess mortality more accurately. Our intervention forms a platform for wider service development in this area both in the inpatient setting and beyond.

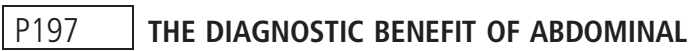 ULTRASOUND SCANS IN INCIDENTALLY ABNORMAL LIVER FUNCTION TESTS}

${ }^{1}$ lain Macpherson*, ${ }^{2}$ Jennifer Nobes, ${ }^{2}$ Ellie Dow, ${ }^{1} J o h n$ Dillon. ${ }^{1}$ Gut Group, University of Dundee; ${ }^{2}$ Department of Blood Sciences, NHS Tayside

\subsection{6/gutjnl-2020-bsgcampus.272}

Introduction An abdominal ultrasound scan (AUSS) is widely recommended in national and international guidelines for the investigation of abnormal liver function tests (LFTs). Abnormal LFTs exist in around 20\% of cases in primary care, placing significant demand on radiology services. However, the evidence for performing AUSS in patients with incidentally abnormal LFTs is weak.

Non-invasive scoring systems such as the NAFLD fibrosis score (NFS) and Fibrosis-4 index (FIB4) are used in the assessment of liver fibrosis. Abnormal results prompt referral to secondary care for further review. It is unclear whether AUSS provides additional diagnostic information in such cases.

Intelligent liver function testing (iLFT) was launched in NHS Tayside in 2018. General Practitioners (GPs) provide relevant clinical details and those with abnormal LFTs have reflex tests without further venepuncture. Non-invasive fibrosis scores are calculated automatically. Management plans with recommended outcomes are then provided: secondary care referral; primary care follow-up; or further investigations and referral criteria.

Methods A retrospective analysis was performed of all patients who had iLFT performed between August 2018 and August 2019, and who had abnormal NFS ( $\geq-1.455$ for patients aged under $65 ; \geq 0.12$ in patients aged 65 or over) and/or FIB4 $(\geq 1.45)$. The result of their ultrasound was documented and its impact on their diagnostic journey recorded.
Diagnosis at Ultrasound in patients with elevated NFS or FIB4

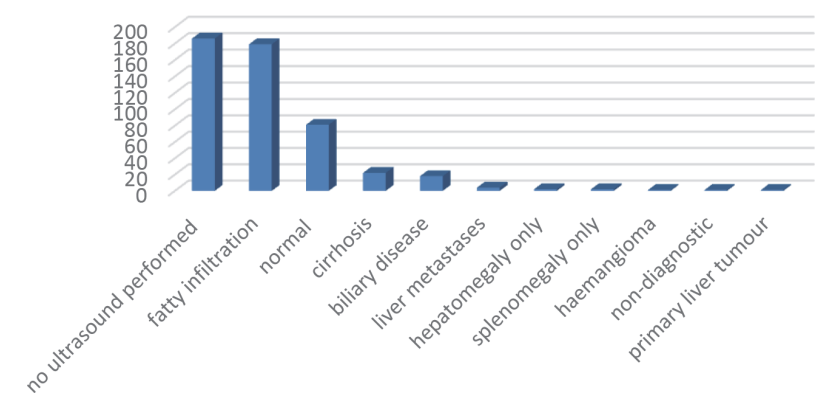

Abstract P197 Figure 1 Diagnosis at ultrasound in patients with elevated NFS and/or FIB-4

Results 497 patients had an iLFT outcome with abnormal NFS and/or FIB4.

311 (62.6\%) had AUSS either prior or parallel to referral. 81 patients had a normal AUSS. 179 (57.6\%) patients had simple fatty infiltration of the liver. 22 (7\%) had confirmatory radiological features of cirrhosis. Overall, AUSS did not add any diagnostic information or alter the clinical pathway in 306 of the 311 patients (98.4\%).

4 patients $(1.3 \%)$ were diagnosed with metastatic disease and 1 patient had a primary liver tumour (neuroendocrine aetiology on subsequent biopsy). All 5 of these patients had co-existent symptoms which would have separately prompted investigations other than AUSS (iron deficiency anaemia in 4 patients, unexplained abdominal pain in 1 patient).

The diagnoses are shown in figure 1.

Conclusions AUSS can play an important role in the diagnosis of biliary disease or malignancy in the setting of symptomatic abnormal LFTs or abdominal pain, but provides little diagnostic benefit in the diagnosis of asymptomatic or incidentally abnormal LFTs. Removing routine AUSS from diagnostic pathways could save significant time and money for radiology departments and GPs, while safely ensuring malignancy is not missed.

\section{P198 SOCIO-ECONOMIC DEPRIVATION ADVERSELY AFFECTS SURVIVAL FOLLOWING LIVER TRANSPLANTATION}

${ }^{1}$ Tom Manship*, ${ }^{1}$ Andrew Robertson, ${ }^{2}$ Emma Robinson, ${ }^{1}$ Kenneth Simpson. ${ }^{1}$ NHS Lothian, Edinburgh, UK; ${ }^{2}$ NHS Tayside, Dundee, UK

\subsection{6/gutjnl-2020-bsgcampus.273}

Introduction Liver transplantation is the only effective treatment for end stage liver disease. It is unclear how socio-economic deprivation affects survival in patients with severe liver disease both transplanted and not listed for transplant.

Methods All notes for adult patients assessed for liver transplantation in Scotland between January 2009 and December 2017 were reviewed. Patients with incomplete data, those who died before transplant, were listed for dual transplant or who were removed from the list were excluded $(n=1045)$. We assessed survival using Kaplan-Meier curves to compare those transplanted to those not listed. Patients not listed as they had a hepatocellular carcinoma outside criteria, or were too well or too unwell to be listed were excluded from the relevant analyses to lower the risk of bias $(n=253)$. We also assessed 
Kaplan-Meier curves showing survival following liver transplantation in SIMD 1 and SIMD 5

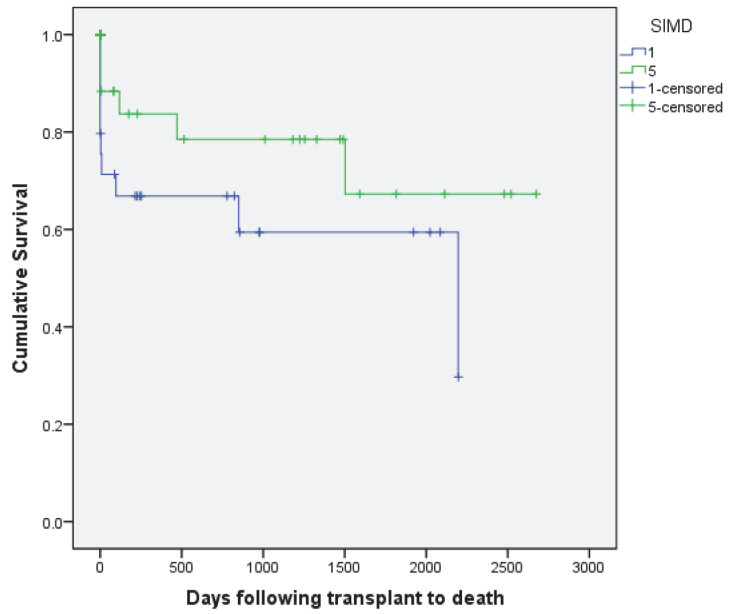

Abstract P198 Figure 1 Kaplan-Meier curves showing survival following liver transplantation in SIMD 1 and SIMD 5

the effect of socio-economic deprivation by comparing survival in the most and least deprived groups in the transplanted and not listed groups. A significance level of $\mathrm{p}<0.05$ was used with the Log rank test.

Deprivation was assessed using the Scottish Index of Multiple Deprivation (SIMD) and groups were paired for analysis (group 1 most deprived; group 5 least deprived)

Results When including all patients those transplanted ( $n=562 ; 103$ deaths) had a significantly better survival than those not listed $(\mathrm{n}=230 ; 139$ deaths) (Mean survival 2219 days (95\% CI 1912-2526) vs. mean survival 645 days $(95 \%$ CI 563-726). (Log rank $\mathrm{p}<0.001)$.

There was no difference in survival when comparing the most deprived to the least deprived (SIMD $1 \quad(n=84 ; 56$ deaths) vs. SIMD $5(\mathrm{n}=32 ; 16$ deaths) $)$ in those patients not listed for transplant. (Mean survival 658 days (95\% CI 474842 ) vs. mean survival 680 days (95\% CI 367-994). (Log rank $\mathrm{p}=0.969$ ).

When comparing survival in the most deprived $(n=133 ; 32$ deaths) to the least deprived $(n=86 ; 13$ deaths) in those patients that were transplanted, patients from the more deprived areas had a poorer survival. (Mean survival 1373 days (95\% CI 1027-1719) vs. mean survival 1998 days (95\% CI 1596-2400) (Log rank $\mathrm{p}=0.046)$.(Figure 1).

Conclusions Overall liver transplantation gives a significant survival advantage compared to those not listed. Patients from more affluent areas of Scotland have improved survival to those from less affluent areas when transplanted. No difference is seen in those patients not transplanted.

\section{P199 IMPACT OF A DISCHARGE PROFORMA ON THE MANAGEMENT OF PATIENTS WITH DECOMPENSATED LIVER DISEASE}

${ }^{1}$ Holly Fraser, ${ }^{2}$ Stuart McPherson, 'Dina Mansour*. 'Queen Elizabeth Hospital, Gateshead, Newcastle Upon Tyne, UK; ${ }^{2}$ Royal Victoria Infirmary, Newcastle upon Tyne, UK

\subsection{6/gutjnl-2020-bsgcampus. 274}

Objectives To produce and implement a discharge proforma, with the aim of improving record keeping and management of patients with decompensated cirrhosis. Decompensated liver disease is associated with a high mortality and can manifest in a variety of manners; thus it is important that certain aspects of these patients' admissions are clearly documented and easily accessible.

Design Discharge letters of 61 patients with decompensated liver disease who had been admitted to QEH between 2017 and 2018 were reviewed and compared to a proforma developed by the gastroenterology team at the RVI (with permission). A similar discharge proforma was developed and integrated for use on the gastroenterology ward at the Queen Elizabeth Hospital, and subsequent discharge letters in 2019 were audited (further 27 letters).

Results The implementation of a discharge proforma enhanced documentation of key features of patient admissions for decompensated cirrhosis. In the 2017-2018 letters $45.0 \%$ of key features were documented; this rose to $67.0 \%$ in the 2019 letters following implementation of the proforma. In addition, there was a $44 \%$ reduction in the number of inpatient deaths and 55\% and 64\% reduction in readmissions (within 30 and 90 days, respectively), following the introduction of the discharge proforma.

Areas which remain poorly documented despite the proforma include renal function, Child Pugh score/prognosis and variceal grading.

Renal function documentation improved marginally with 9 of the 2019 patient letters containing documentation of renal function, as opposed to the 0 letters across 2017 and 2018; however this still only equates to $33.3 \%$ of the discharge proformas containing information about patients' renal function. Child Pugh score was not documented for any of the 2019 cohort and the grade of varices was documented in just one of the discharge proformas (16.7\% of 2019 cohort), which is a $20.8 \%$ and $10.6 \%$ reduction compared to the 2017 and 2018 discharge letters.

Conclusions Implementation of a discharge proforma for decompensated liver disease patients is associated with fewer inpatient deaths, a reduction in readmissions and improved documentation in their discharge letters. Further work is required with the implementation of the proforma, particularly around education regarding its use, to ensure it is utilised appropriately. A similar project is being undertaken at the RVI, with the aim of producing a proforma for the British Society of Gastroenterologists.

\section{P200 REAL-LIFE COMPARISON OF TRANSIENT ELASTOGRAPHY (FIBROSCAN ${ }^{\circledR}$ ) TO LIVER BIOPSIES: A UK DISTRICT GENERAL HOSPITAL EXPERIENCE}

${ }^{1}$ Georgios Marinopoulos*, 'Maja Kopczynska, 'Bethany Miller, ${ }^{2}$ Mahesh Bhalme. 'Salford Royal NHS Foundation Trust, Manchester, UK; ${ }^{2}$ Bolton NHS Foundation Trust, Bolton, UK

\subsection{6/gutjnl-2020-bsgcampus.275}

Background and Aim Over the last decade, the development of liver stiffness measurement using transient elastography (FibroScan ${ }^{\circledR}$ ) and its widespread use has become the standard for non-invasive staging of liver fibrosis. Our study explores a real-life experience on concordance of fibrosis staging between the fibroscan and the liver biopsies aiming to assess its reliability.

Method We conducted a retrospective study between September 2014 and May 2019 comparing fibroscan and liver biopsy findings. The FibroScan 502 Touch results were interpreted to 